\title{
Corona discharge of a vibrated insulating box with granular medium
}

\author{
Laurent Schoenau ${ }^{1} \cdot$ Tobias Steinpilz $^{1} \cdot$ Jens $_{\text {Teiser }}{ }^{1} \cdot$ Gerhard Wurm $^{1}$ (I)
}

Received: 16 February 2021 / Accepted: 29 May 2021 / Published online: 7 July 2021

(c) The Author(s) 2021

\begin{abstract}
Corona discharges are luminous signs of strong local electric fields allowing a continuous discharge into the surrounding atmosphere. They commonly occur at the ends of conductors at high voltage. Here we report the observation of a faint glow surrounding an insulating $\mathrm{cm}$-sized box filled with mm-sized basalt beads. At an ambient pressure in the mbar range two light bands occur as soon as and only if the box is vibrated and only if it is filled with a granular medium. In addition, a glow also occurs at the inside of the box. We measured periodic electric fields at the outside of the box with spatial peaks at the positions of the light bands. The period correlates to the vibration frequency. These observations imply strong alternating fields beyond atmospheric breakdown, which are generated inside and also emerge at the outside of the insulating box. The observations can be explained by tribocharging and periodic displacement of charges between grains and the inside walls of the box.
\end{abstract}

Keywords Collisional charging $\cdot$ Tribocharging $\cdot$ Corona discharge

\section{Introduction}

Charging of colliding grains is a widely known phenomenon. In fact, charge separation is hard to prevent, if two surfaces get in contact and separate again [8]. For two different insulating materials, there is a bias in the direction of charge transfer, i.e. which material charges negative and which positive along the triboelectric series $[9,10]$.

However, charging can proceed only to a certain level. Tribocharging itself might be limited by its underlying processes but charging also competes with discharge $[4,11$, 14]. If the setting is insulated enough that discharge by conduction does not occur, and if charge separation continued, there would still be an upper limit of charging. Electrical fields would become large enough for breakdown of the surrounding atmospheric gas, if present [12]. These can be large scale discharges, visible as lightning like in a thunderstorm or volcano eruption $[1,13]$. It can be discharges on a smaller scale between grains, which might or might not be visible by producing radiation $[7,11,14]$. Low atmospheric pressure is

Gerhard Wurm

gerhard.wurm@uni-due.de

1 Faculty of Physics, University of Duisburg-Essen, Lotharstr. 1, D-47057 Duisburg, Germany important in this context as it reduces necessary breakdown voltages, at least down to a certain pressure [2, 3, 14].

Therefore, luminous discharges are common in granular media or granular gases. However, in these cases discharges occur between grains. This is not the case in the experiments reported here, where the discharge takes place at the outside of a well insulating box, while all grains are hidden inside the container.

We report a glow which occurs within an agitated granular medium but which, in addition, is also surrounding the insulating box that holds the granular medium. We argue that this is generated by AC Corona discharges driven by triboelectric fields produced by the vibrations of the granular medium.

\section{Experiment}

Figure 1 shows a sketch of the experiment.

A box with the inside volume of 36 by 36 by $36 \mathrm{~mm}$ and a wall thickness of $2 \mathrm{~mm}$ made of ABS (AcrylnitrilButadien-Styrol-Copolymer) or PET (Polyethylene terephthalate) is shaken by a voice coil. One side of the box is a glass window. As the view through the window does not allow a direct statement if a glow is on the outside or inside, a control experiment was conducted with observation from 
Fig. 1 Left: Sketch of the basic setup of the experiments; Right: Image of a box filled with basalt beads
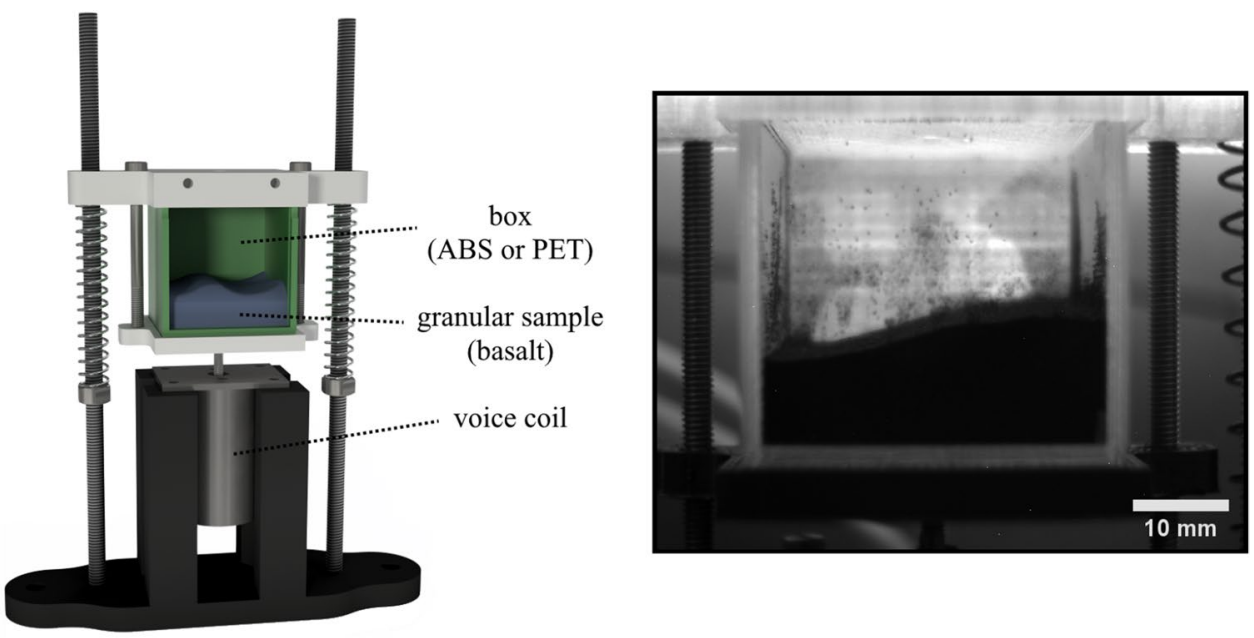

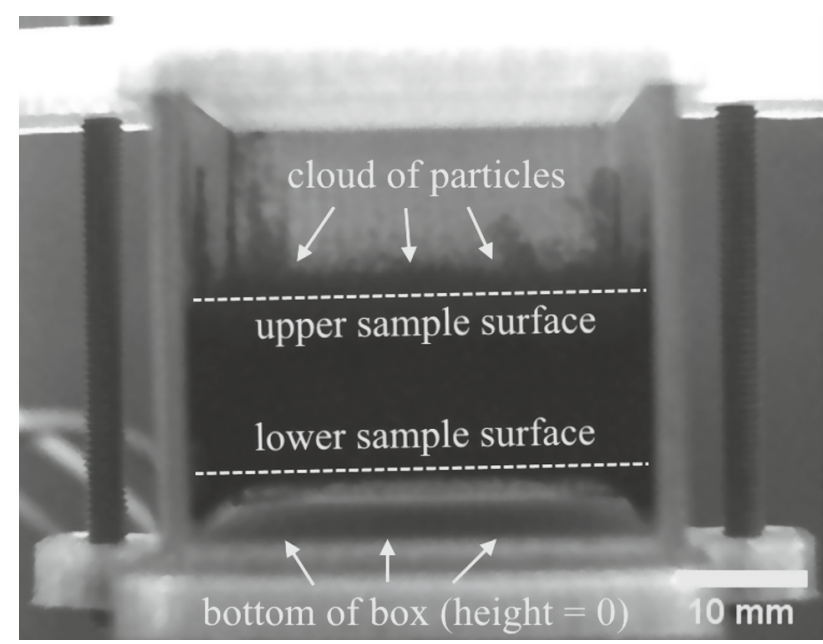

Fig. 2 Snapshot of a sample during vibration

above. Here, the box was somewhat higher to avoid spilling of grains out of the box during the agitation. The boxes were filled to about $1.5 \mathrm{~cm}$ in height with basalt spheres of $165 \mu \mathrm{m}$ $\pm 15 \mu \mathrm{m}$ diameter. During shaking the sample remained rather compact with a clear lower and upper surface and a cloud of particles on top as seen in Fig. 2. It therefore resembles a granular fluid with kind of a granular gas on top.

The whole setup is placed within a vacuum chamber which is electrically connected to ground. A cooled CCD camera with attached f1.4 $25 \mathrm{~mm}$ optics is connected to a viewport of the vacuum chamber with a light-tight baffle to detect any light originating within. Each frame has an exposure time of $60 \mathrm{~s}$.

In addition, electrical fields were probed at the sides of the vibrating box by a needle attached to an oscilloscope with the setup being outside of the vacuum chamber ( 1 bar). In both cases the chamber was vibrated with $17 \mathrm{~Hz}$ where the observed glow was most intense (without detailed analysis).

\section{Observations and electrical field measurements}

Our core measurements were images (exposure time: 60 s) of the inside of the vacuum chamber at varying atmospheric pressure. Depending on the pressure, a glow of certain intensity could be detected. For a quantitative analysis the images were corrected for the read-out bias, the dark current and hot and cold pixels. Afterwards the total brightness of each image (front view only) is calculated as a proxy for the glow's intensity.

\subsection{Basic glow features}

Figure 3 shows the spatial distribution of light for the two observation geometries used. The top image gives a top view, showing glow surrounding the sample container, but also occurring above the sample, with reduced intensity. The glow mainly is distributed close to the walls of the box.

The bottom panel of Fig. 3 shows the front view, where the glow on the outside splits up into two light bands. The bottom light band corresponds to a region at the bottom of the sample container. There are some variations but, in general, the top light band roughly corresponds to the surface region of the compact part of the sample (granular fluid). We tested different depths of the granular bed. While we did not further quantified the positions, the top light band shifted upwards for large sample depths in agreement of tracing the surface layer. This spatial glow profile implies a relation between glow, walls and top and bottom of the granular sample. The glow is not limited in time. While there is some variation over time once the vibrations are started, there is especially no time at which 


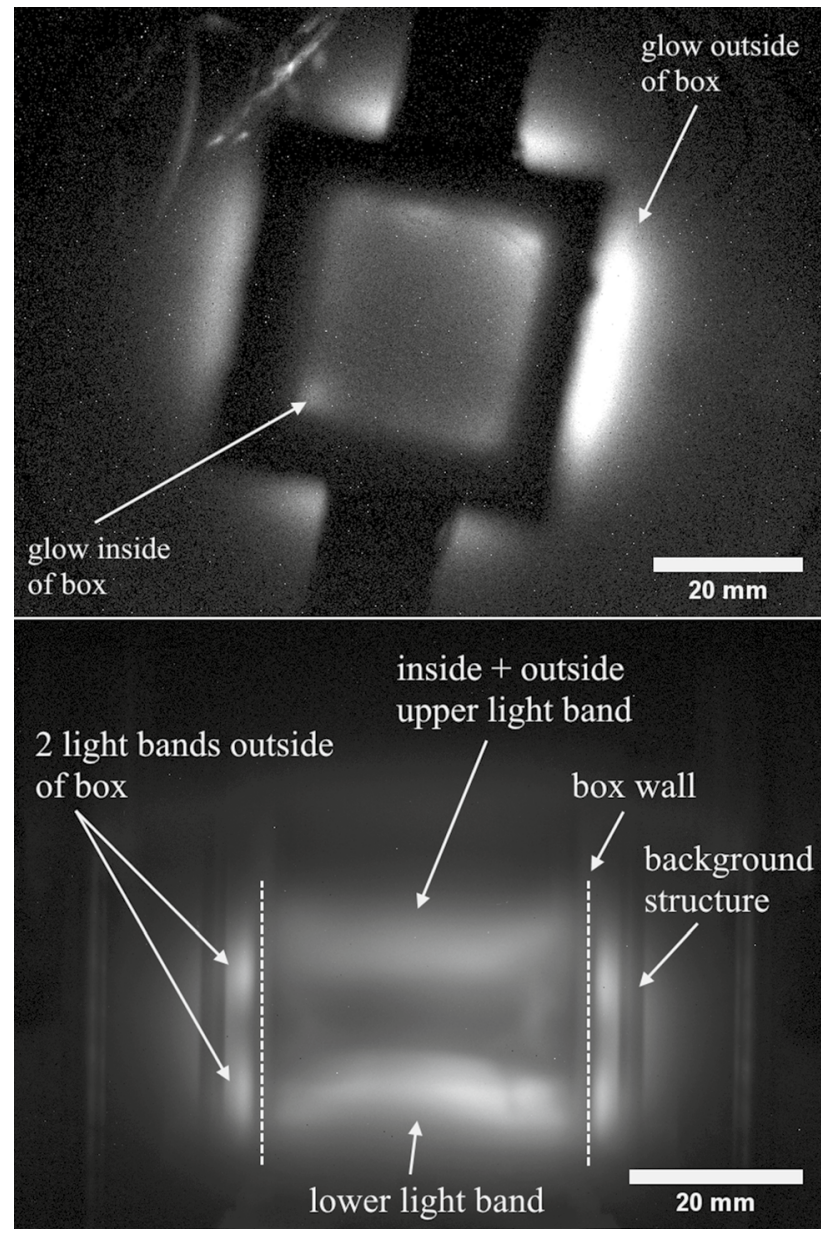

Fig. 3 Top: Glow in top view of an open box. The dark frame is the box itself and its mount; Bottom: Front view of the glow (corrected for hot pixels and dark current). The two light bands correspond to the bottom and the top of the compact part of the granular sample. Both images are enhanced in contrast for better visibility (top view by a factor of 10 more in comparison to the front view)

no glow is observed. As long as the container is vibrated, it glows. The longest observation lasted 50 minutes, limited only by a maximum temperature of the voice coil, which heats up at low pressure due to loss of air cooling.

\subsection{Pressure dependence of glow}

The intensity of the glow strongly depends on the pressure as seen in Fig. 4. Only the images of the front view were used for this analysis. The intensity of the glow has a maximum at about 0.5 mbar. The intensity of the glow decreases to smaller and higher pressures. This implies a gas breakdown nature of the glow. Some estimates on a Paschen breakdown are given below and the pressure range given is in favor of breakdown as explanation. However, we do not aim to model this in detail here. To guide the eye, we fitted the brightness

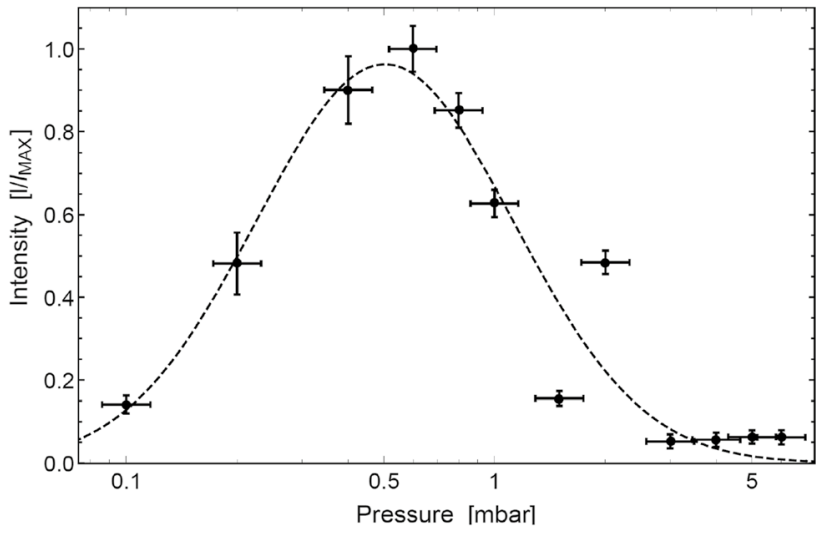

Fig. 4 Pressure dependence of total brightness of a glow normalized to the maximum

curve by a log-normal distribution though which provides a reasonable agreement to the data.

\subsection{Electrical fields surrounding the box}

If the glow bands are related to a breakdown within the thin atmosphere, the electric fields on the outside of the sample container should be strongest at their positions. We therefore measured the electric field at normal pressure (1000 mbar), where no glow occurs and the field is (likely) not disturbed by discharges. The measured field changes periodically with a frequency perfectly matching the agitation frequency. Figure 5 shows the evolution of the electric field over one period of such a measurement. For a better signalnoise ratio the curve shown is the mean of 100 periods of the agitation cycle.

The voice coil also induces a signal into the probe. This was measured for an empty container. The signal was

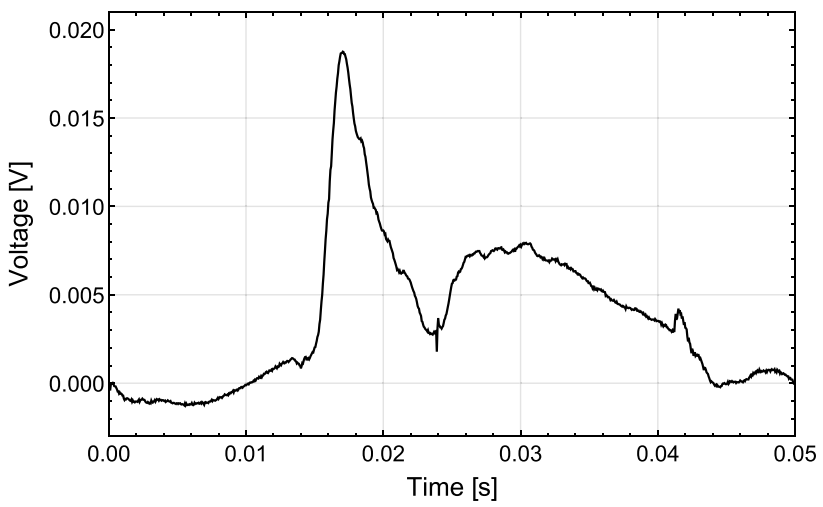

Fig. 5 Temporal evolution of the periodic electrical field. For a better signal-noise ratio the curve is a mean of 100 periods showing only one period of the agitation cycle. The signal for the vibrating box without granular sample is already subtracted 
much smaller, had a different shape and was subtracted from the measurements with the granular sample. We did not attempt to attribute absolute field measures to our simple probe measurements. Assuming that no discharges occur at $1 \mathrm{bar}$, the field does not trace the exact situation at low pressure, but we still see the increase and decrease of the field as suggested by the model below.

The maximum field strength varies with height from the bottom of the box as seen in Fig. 6. The positions of the maxima of the electric fields are consistent with top and bottom of the compact part of the granular sample, also considering the vertical motion of the sample by a few millimeters (which will widen the half-widths of the two peaks). The maxima also correspond well to the positions of the light bands.

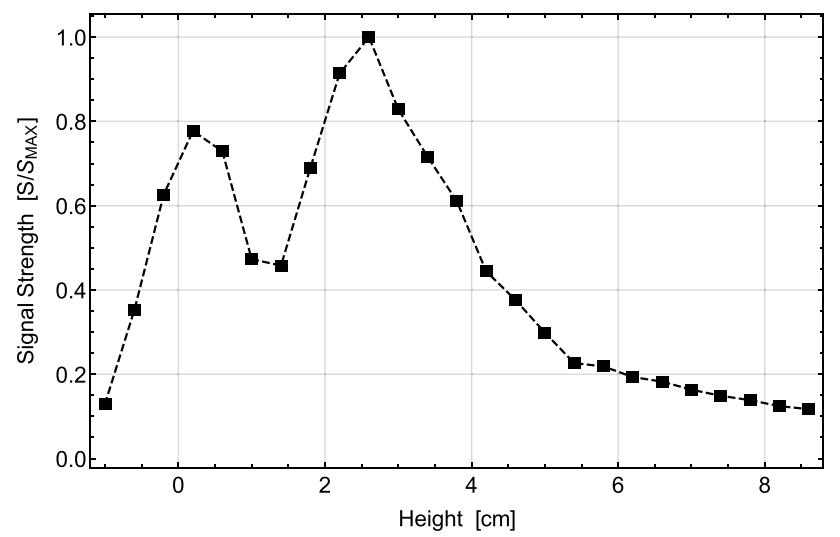

Fig. 6 Height dependence of the maximum electrical field. Height zero corresponds to the bottom of the box. The positions of the maxima at about $0.5 \mathrm{~cm}$ and $2.5 \mathrm{~cm}$ correspond to the lower and upper sample surface as seen in Fig 2

\section{Glow discharge model}

The data give very clear evidence that the vibrations of the granular medium induce electric fields in certain locations. These are strong enough to lead to breakdown at low pressure. The electrical field has to be alternating. This way a continuous glow or what we call corona discharge can occur. Tribocharging to an equilibrium charge would not explain continuous glow. Though fields are generated inside of the box and though one might expect the highest fields there, discharging has to be suppressed on the inside to enable breakdown at the outside.

[15] find pattern formations of charges in simulations of vibrated samples. They also suggest states where the charge oscillates. This would be one choice of explanation. However, as the center of the sample (in top view) is glowing rather weak and with glow only being restricted to two bands at the top and bottom of the dust, collisions between particles and the wall seem to be most plausible and more simple as argument as collisions between different materials will always lead to charging. [9] e.g. consider this as main driver for charging of a vibrated granular bed in a similar basic setup but with acrylic glass as walls and glass beads as sample. In this case, it has to be explained though, why a continuous discharge occurs. Charging between two different materials (basalt and ABS) is biased and does not produce alternating fields. Fig. 7 is a model that potentially explains the observed behaviour as sequence of events for one period.

We start by assuming that tribocharging charges the inside of the wall to an equilibrium charge along the height of the sample. Without loss of generality we assume the inner wall to charge positive, grains negative. The electric fields important here are then generally produced by displacement of the bulk of grains with respect to the wall. A sequence of events for one period is then as follows.
Fig. 7 Discharge and glow model. Displayed is a sketch at the wall on one side at different times through one vibration cycle. The particles and the wall charge with different sign and form a capacitor. Agitation shifts the particles with respect to the wall and leads to charge offset at the top and at the bottom of the sample. Electrical fields form, strong enough for breakdown in a thin atmosphere

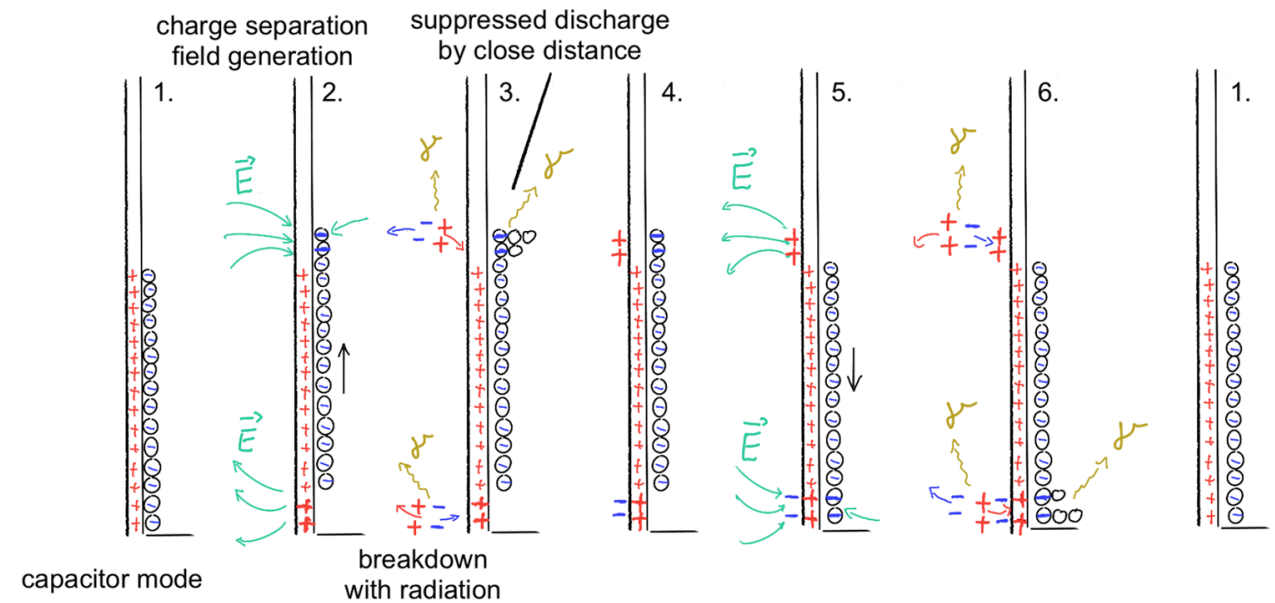


1. Collisions between grains and wall charge the wall positive in the first step. Therefore, a wall charged positive on the inside is right next to a negative 'wall' of grains. There is no electrical field on the outside of the box in this situation as both, wall and grains, act like a capacitor.

2. The vibration now lifts the granular sample. This shifts the negative particle wall upwards and shifts both walls relative to each other. The center part is now still like a capacitor and there is no field in the center part on the outside of the container. However, at the top and bottom, net charges induce electric fields.

3. These fields get strong enough to allow breakdown discharge in the atmosphere at the outside and top and bottom part neutralize. There are also breakdowns at the inside, but due to the particle sample, this requires situations, where grains are not too close to the wall and each other. Otherwise, the close distance does not allow discharge as no Townsend avalanche can develop.

4. After discharge on the outside, charges also sit on the outside of the wall but otherwise there is no more external field.

5. The sample drops back but as it does, the charge at the top and bottom is again off balance and a now reversed field is generated on the outside.

6. A second breakdown occurs to restore the charge balance and sets the situation back to the original capacitor mode.

In a certain sense, this is the basic process occuring in a sliding mode triboelectric nanogenerator but with a granular medium and without generator stage electrodes [5].

\subsection{Breakdown discharge estimates}

In order to estimate if the assumption of an electric breakdown is plausible, we can estimate the electric field generated by the charges and compare it's strength to the field needed for breakdown. As approximation, we consider the top and bottom e.g. in configuration 2 or 5 in Fig. 7 to be extended surfaces of constant charge density with a constant electric field close to the surface. The further away, the weaker the field though, which implies that the breakdown is mostly occuring close to the surface. In any case, the electric field needed for breakdown in a capacitor configuration in view of Townsend electron avalanches is the Paschen law:

$E=\frac{C_{2} \cdot P}{\ln \left(C_{1} \cdot P \cdot d\right)-\ln \left(\ln \left(1+\frac{1}{\gamma}\right)\right)}$.

For air the constants are $C_{1}=10.95(\mathrm{~Pa} \cdot \mathrm{m})^{-1}$ and $C_{2}=273.8 \mathrm{~V} /(\mathrm{Pa} \cdot \mathrm{m})$. It is $d$ the distance between the electrodes and $\gamma$ the probability that an ion impacting a surface releases a new electron which we assume to be $\gamma=1$ here. The electric field of a single plate with charge density $\sigma$ is half the field of a capacitor or [6]:

$E=\frac{\sigma}{2 \epsilon_{0}}$,

with the dielectric constant $\epsilon_{0}$. The needed surface charge density then becomes:

$\sigma=\frac{2 \cdot \epsilon_{0} \cdot C_{2} \cdot P}{\ln \left(C_{1} \cdot P \cdot d\right)-\ln \left(\ln \left(1+\frac{1}{\gamma}\right)\right)}$.

We did not specify the distance $d$ yet as we do not have a real plate capacitor. We consider the following two situations. On the outside, any boundary is very distant, e.g. $d=0.1 \mathrm{~m}$. Inserting values, the critical surface density at 1 mbar would then be $\sigma=10^{-7} \mathrm{C} / \mathrm{m}^{2}$. For one layer of grains of the given size that corresponds to about 16.000 charges on each 165 $\mu m$ grain. If we assume that basalt grains accumulate as much charge in contact with the plastic walls as among each other but preferentially with one polarity, data e.g. from [14] would suggest a typical charge of $10 \mathrm{pC}$ or more than a million charges per grain scaling by particle size squared. This is orders of magnitudes more than needed for breakdown, so a breakdown on the outside is very plausible at low pressure even with some dielectric material in between.

On the other side assuming a distance $d=330 \mu \mathrm{m}$ that corresponds to the spacing between grains on the order of the grain diameter, Eq. 3 becomes negative and no longer applies, i.e. an electron avalanche cannot develop. Only once the distance between grains increases to about $650 \mu \mathrm{m}$ discharge would be possible again but the necessary charge for breakdown would be on the order of the observed basalt charges in [14] of millions or larger. We note that this is clearly not a plate capacitor configuration but consider this approximation as suitable estimate here. In any case, it is plausible then that discharges above the grain bed can occur but they are suppressed on the inside of the bulk of the granular sample, with grains in close proximity.

Equation 1 also suggests that the necessary charge density for discharge increases with pressure and, therefore, the glow decreases in intensity in agreement to Fig. 4. At low pressure, the electric field over longer and longer distances becomes important as the mean free path of the gas molecules increases. Here, it becomes increasingly important that the electric field decreases with distance to the wall as our configuration gets less and less capacitor like then. It is also plausible then that the luminosity decreases to lower pressure. This implies a maximum of the light intensity at a certain pressure in the mbar range in agreement to the observations. 


\section{Conclusion}

Somewhat unexpectedly, we observed a vibrated insulating box holding a granular medium to glow at low pressure in what we would call a corona discharge. This can well be explained by tribocharging between grains and wall. That breakdown rather occurs on the outside than on the inside is sign of an increased breakdown voltage of a dense granular medium, as distances between grains and grains and wall are just too short for electron avalanches to develop at the low pressure studied.

Funding Open Access funding enabled and organized by Projekt DEAL.

\section{Compliance with ethical standards}

Conflict of interest The authors declare that they have no conflict of interest.

Open Access This article is licensed under a Creative Commons Attribution 4.0 International License, which permits use, sharing, adaptation, distribution and reproduction in any medium or format, as long as you give appropriate credit to the original author(s) and the source, provide a link to the Creative Commons licence, and indicate if changes were made. The images or other third party material in this article are included in the article's Creative Commons licence, unless indicated otherwise in a credit line to the material. If material is not included in the article's Creative Commons licence and your intended use is not permitted by statutory regulation or exceeds the permitted use, you will need to obtain permission directly from the copyright holder. To view a copy of this licence, visit http://creativecommons.org/licenses/by/4.0/.

\section{References}

1. Cimarelli, C., Alatorre-Ibargüengoitia, M.A., Aizawa, K., Yokoo, A., Díaz-Marina, A., Iguchi, M., Dingwell, D.B.: Multiparametric observation of volcanic lightning: Sakurajima Volcano. Japan Geophys. Res. Lett. 43(9), 4221-4228 (2016). https://doi.org/10. 1002/2015GL067445

2. Eden, H.F., Vonnegut, B.: Electrical breakdown caused by dust motion in low-pressure atmospheres: considerations for mars. Science 180(4089), 962-963 (1973). https://doi.org/10.1126/science. 180.4089 .962
3. Farrell, W.M., McLain, J.L., Marshall, J.R., Wang, A.: Will the mars helicopter induce local martian atmospheric breakdown? Planet Sci. J. 2, 46 (2021)

4. Haeberle, J., Schella, A., Sperl, M., Schröter, M., Born, P.: Double origin of stochastic granular tribocharging. Soft matter 14(24), 4987-4995 (2018)

5. He, W., Liu, W., Chen, J., Wang, Z., Liu, Y., Pu, X., Yang, H., Tang, Q., Yang, H., Guo, H., Hu, C.: Boosting output performance of sliding mode triboelectric nanogenerator by charge space-accumulation effect. Nat. Commun. 11, 4277 (2020). https://doi.org/ 10.1038/s41467-020-18086-4

6. Jackson, J.D.: Classic. Electrodyn., 3rd edn. John Wiley, Hoboken, N. H. (1999)

7. Krauss, C.E., Horányi, M., Robertson, S.: Modeling the formation of electrostatic discharges on Mars. J. Geophys. Res. (Planets) 111(E2), E02001 (2006). https://doi.org/10.1029/2004JE002313

8. Lacks, D.J., Shinbrot, T.: Long-standing and unresolved issues in triboelectric charging. Nat. Rev. Chem. 3(8), 465-476 (2019)

9. Liao, C.C., Hsiau, S.S., Huang, T.Y.: The effect of vibrating conditions on the electrostatic charge in a vertical vibrating granular bed. Powder Technol. 208(1), 1-6 (2011). https://doi.org/10. 1016/j.powtec.2010.10.021

10. Lowell, J., Rose-Innes, A.: Contact electrification. Adv. Phys. 29(6), 947-1023 (1980). https://doi.org/10.1080/0001873800 0101466

11. Méndez Harper, J., Dufek, J.: The effects of dynamics on the triboelectrification of volcanic ash. J. Geophys. Res. (Atmospheres) 121(14), 8209-8228 (2016). https://doi.org/10.1002/2015JD0242 75

12. Méndez Harper, J., Dufek, J., McDonald, G.D.: Detection of spark discharges in an agitated Mars dust simulant isolated from foreign surfaces. Icarus 357, (2021). https://doi.org/10.1016/j.icarus.2020. 114268

13. Saunders, C.: Charge separation mechanisms in clouds. Space Sci. Rev. 137(1-4), 335-353 (2008). https://doi.org/10.1007/ s11214-008-9345-0

14. Wurm, G., Schmidt, L., Steinpilz, T., Boden, L., Teiser, J.: A challenge for martian lightning: limits of collisional charging at low pressure. Icarus 331, 103-109 (2019). https://doi.org/10.1016/j. icarus.2019.05.004

15. Yoshimatsu, R., Araújo, N.A.M., Wurm, G., Herrmann, H.J., Shinbrot, T.: Self-charging of identical grains in the absence of an external field. Sci. Rep. 7, 39996 (2017). https://doi.org/10. 1038/srep39996

Publisher's Note Springer Nature remains neutral with regard to jurisdictional claims in published maps and institutional affiliations. 